\title{
The characteristics of bacterial nanocellulose gel releasing silk sericin for facial treatment
}

\author{
Pornanong Aramwit ${ }^{*}$ and Nipaporn Bang
}

\begin{abstract}
Background: Recently, naturally derived facial masks with beneficial biological properties have received increasing interest. In this study, silk sericin-releasing bacterial nanocellulose gel was developed to be applied as a bioactive mask for facial treatment.

Results: The silk sericin-releasing bacterial nanocellulose gel produced at a pH of 4.5 had an ultrafine and extremely pure fiber network structure. The mechanical properties and moisture absorption ability of the gel were improved, compared to those of the commercially available paper mask. Silk sericin could be control-released from the gel. A peel test with porcine skin showed that the gel was less adhesive than the commercially available paper mask, which would be removed from the face more easily without pain. The in vitro cytotoxicity test showed that the gel was not toxic to L929 mouse fibroblast and HaCaT human keratinocyte cells. Furthermore, when implanted subcutaneously and evaluated according to ISO10993-6 standard, the gel was not irritant to tissue.
\end{abstract}

Conclusion: The silk sericin-releasing bacterial nanocellulose gel had appropriate physical and biological properties and safety for the facial treatment application.

Keywords: Bacterial nanocellulose, Gluconacetobacter xylinus, Coconut water, Silk sericin, Facial mask

\section{Background}

Nowadays, various kinds of facial masks are applied for different purposes: for example, revitalizing, healing, and refreshing, and may yield other temporary or long-term benefits to facial skin. Recently, searching for a new naturally derived facial mask with beneficial biological properties has become highly competitive. Cellulose is an organic polysaccharide consisting of a linear chain of several hundred to over ten thousand $\beta(1 \rightarrow 4)$ linked $D$-glucose units [1]. Cellulose is frequently derived from plant sources; however, it can also be synthesized by a variety of microorganisms such as bacteria, algae, and fungi [2]. Biosynthetic bacterial cellulose shows appealing characteristics of high purity and unique physicochemical properties such as high tensile strength, crystallinity, and water absorption capacity [3]. Gluconacetobacter xylinus (formerly known as Acetobacter xylinum) is a species of gram-negative bacteria that can

\footnotetext{
* Correspondence: aramwit@gmail.com

Bioactive Resources for Innovative Clinical Applications Research Unit and Department of Pharmacy Practice, Faculty of Pharmaceutical Sciences,

Chulalongkorn University, PhayaThai Road, Phatumwan, Bangkok 10330, Thailand
}

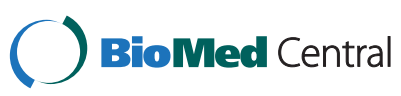

(c) 2014 Aramwit and Bang; licensee BioMed Central Ltd. This is an Open Access article distributed under the terms of the Creative Commons Attribution License (http://creativecommons.org/licenses/by/4.0), which permits unrestricted use, distribution, and reproduction in any medium, provided the original work is properly credited. The Creative Commons Public Domain Dedication waiver (http://creativecommons.org/publicdomain/zero/1.0/) applies to the data made available in this article, unless otherwise stated. produce high amounts of cellulose. Glucose was used as the common substrate for the bacterial nanocellulose produced by G. xylinus [4]. Therefore, searching for cost-effective sources of glucose that can form bacterial nanocellulose with maintained high purity and quality is important.

Coconut is a fruit grown in tropic and subtropic areas that is known for its great versatility, as seen in the many domestic, commercial, and industrial uses of its different parts. Coconut contains a large quantity of coconut water, which is mostly discarded as waste from various agro industries. Coconut water is rich in sucrose (sugar) and nitrogen-containing compounds. Therefore, it could be a cheap substrate for the production of bacterial nanocellulose [5].

In this study, the bacterial nanocellulose was produced at the surface of coconut water and other suitable media by a gram-negative rod-shaped bacterium, G. xylinus. The fermentation was carried out in static cultures at $30^{\circ} \mathrm{C}$ in acidic $\mathrm{pH}$ varying from 3 to 5 . We investigated the effect of the processing $\mathrm{pH}$ on the formation of bacterial nanocellulose gel. As the aim was to be applied as a bioactive facial mask, our bacterial

ricle unless otherwise stated. 
nanocellulose gel was adsorbed with silk sericin. Silk sericin is a biocompatible protein derived from the silkworm cocoon. The biological roles of silk sericin such as an antioxidant, bioadhesive, and bioactive agent, as well as a promising implant for tissue-supporting prosthetics for the human body, have been reported [6,7]. Silk sericin could also activate collagen production and significantly increase epithelialization in wounds [8-10]. Herein, the physical properties of the silk sericinreleasing bacterial nanocellulose gel produced at optimal $\mathrm{pH}$ were characterized. A test of the release of silk sericin from the gel was carried out. In addition, in vitro cytotoxicity and in vivo safety tests according to ISO 10993-6 standards were performed, in comparison to the commercially available paper mask (Facial Paper Mask, Hefei Wenqi Industrial and Trading, Co., Ltd., Anhui, Republic of China). It is hypothesized that silk sericin-releasing bacterial nanocellulose gel would be a good candidate as a bioactive facial mask for facial treatment. Silk sericin released from bacterial nanocellulose gel may help to revitalize, heal, or refresh the skin.

\section{Results and discussion}

\section{Physical appearance and structure of the bacterial} nanocellulose gel

We first studied the influence of $\mathrm{pH}$ condition on the formation of bacterial nanocellulose gel because it was supposed that $\mathrm{pH}$ may affect the rate of bacterial growth, which was important for the formation of bacterial nanocellulose gel. The bacterial nanocellulose gels prepared from various $\mathrm{pH}$ had different physical appearances, as shown in Figure 1. The gels could be set up at a pH of 3.5 to 5 but did not form at a $\mathrm{pH}$ of 3 . Table 1 summarizes the physical appearance and thickness of the bacterial nanocellulose gels prepared at different $\mathrm{pH}$. At a $\mathrm{pH}$ of 3.5 and 4, hard and stable gels with a smooth surface were produced. The gel produced at a $\mathrm{pH}$ of 4.5 was also stable with a smooth surface but softer. In contrast, the production at a pH of 5 resulted in a very soft gel. The thickness of bacterial nanocellulose gels seemed to decrease with the increasing $\mathrm{pH}$. The processing $\mathrm{pH}$ at 3.5 produced a $0.7 \mathrm{~cm}$ gel while the $\mathrm{pH}$ at 5 produced a $0.3 \mathrm{~cm}$ gel. $\mathrm{A} \mathrm{pH}$ of 4.5 would be the optimal condition for bacterial growth, as previously reported by Embuscado et al. [3]. The
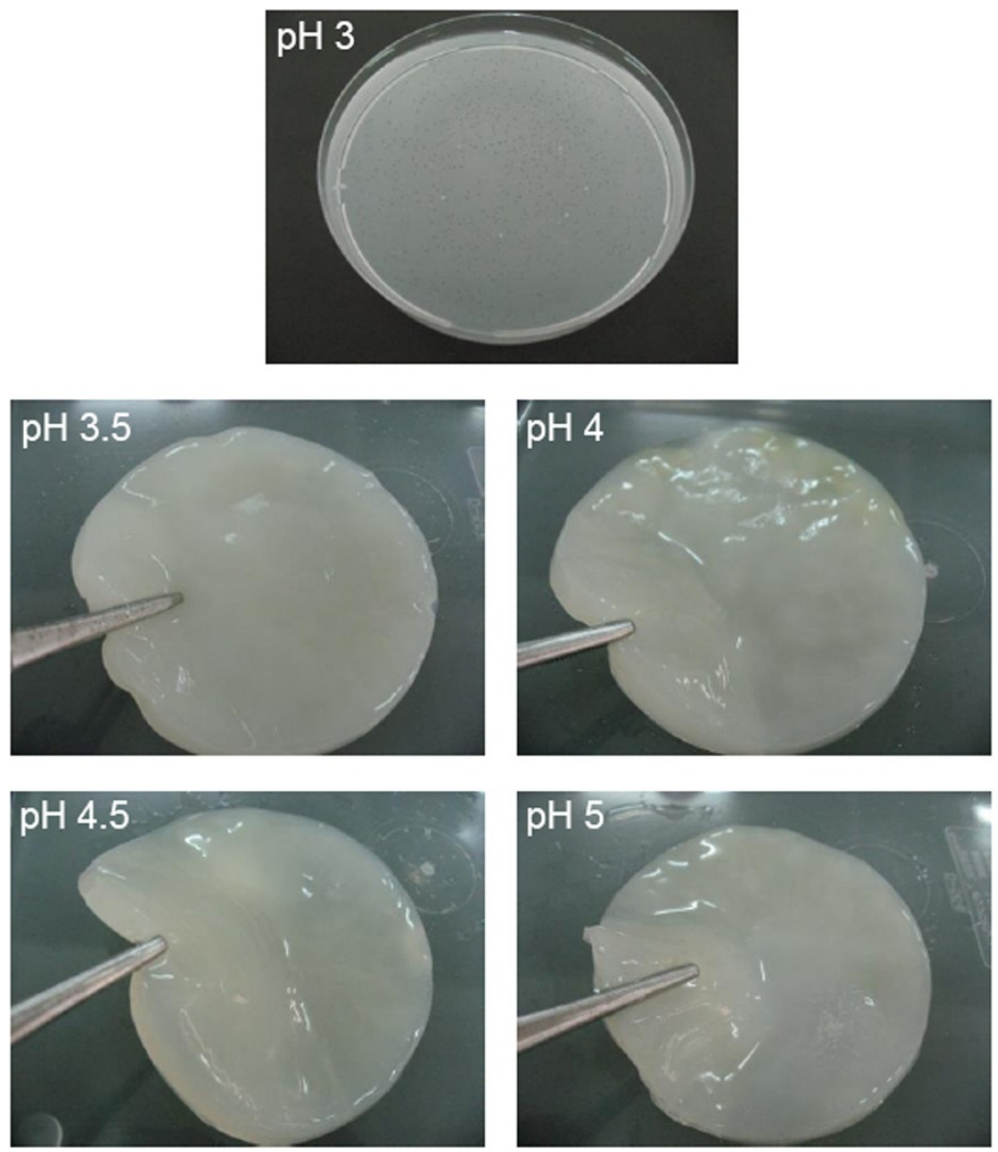

Figure 1 Physical appearance of the bacterial nanocellulose gels prepared at different $\mathrm{pH}(3,3.5,4,4.5$, and 5$)$. 


\begin{tabular}{|c|c|c|}
\hline Processing $\mathrm{pH}$ & $\begin{array}{l}\text { Physical appearance of the } \\
\text { nanocellulose gel }\end{array}$ & $\begin{array}{l}\text { Thickness } \\
(\mathrm{cm})\end{array}$ \\
\hline 3 & Not formed & - \\
\hline 3.5 & Hard and stable gel with smooth surface & 0.7 \\
\hline 4 & Hard and stable gel with smooth surface & 0.5 \\
\hline 4.5 & Soft and stable gel with smooth surface & 0.6 \\
\hline 5 & Very soft gel with smooth surface & 0.3 \\
\hline
\end{tabular}

$0.6 \mathrm{~cm}$ soft and stable gel with a smooth surface produced at a $\mathrm{pH}$ of 4.5 was selected for further investigation in this study. To improve the biological properties, the bacterial nanocellulose gel prepared at a $\mathrm{pH}$ of 4.5 was absorbed with silk sericin solution to obtain the silk sericinreleasing bacterial nanocellulose gel. It is well known that silk sericin has many biological roles such as an antioxidant, bioadhesive, and bioactive activities. It could also activate collagen production and epithelialization, which would be beneficial for facial treatment application [6-10]. The structure of silk sericin-releasing bacterial nanocellulose gel was observed in comparison to the commercially available paper mask, as displayed in Figure 2. We found that the silk sericin-releasing bacterial nanocellulose gel showed a highly fibrous structure with very fine fibers, which corresponded to the report of Vandamme et al. [11] that the bacterial cellulose usually has an ultrafine and extremely pure fiber network structure. On the other hand, the cotton fibers of the commercially available paper mask were much larger $(\sim 10 \mu \mathrm{m}$ in diameter).

\section{Mechanical properties of the silk sericin-releasing bacterial nanocellulose gel}

To be applied as a facial mask, the silk sericin-releasing bacterial nanocellulose gel should have appropriate mechanical properties. For example, it should be flexible, stretchable, and elongatable in order to adhere completely to the whole of the facial skin. The tensile modulus and percentage of elongation of the dried and wet silk sericin-releasing bacterial nanocellulose gel, compared with those of the commercially available paper mask, are demonstrated in Table 2. Both in the dry and wet conditions, the tensile modulus of the silk sericinreleasing bacterial nanocellulose gel were significantly higher than that of the commercially available paper mask. The percentage of elongation of both samples in the dry state was the same. However, in the wet state, the silk sericin-releasing bacterial nanocellulose gel had a significantly higher elongation percentage than the commercially available paper mask. This could be explained by the fact that our gel is a bacterial nanocellulose, which has more crystalline structure and forms characteristic ribbon-like microfibrils, compared to the plant cellulose of a paper mask [12]. In addition, the silk sericin absorbed on the fibers of our bacterial nanocellulose gel would improve the mechanical properties. Improvement of the mechanical properties by surface medication has been reported elsewhere $[13,14]$.

\section{Moisture absorption capability of the silk sericin-releasing bacterial nanocellulose gel}

The facial mask should have good water absorption ability in order to maintain the moisture within its structure during the treatment. Figure $3 \mathrm{~A}$ shows the moisture absorption capability of the silk sericin-releasing bacterial nanocellulose gel, compared to that of the commercially available paper mask. Initially, the amount of water absorbed on the silk sericin-releasing bacterial nanocellulose gel was increased over time and became stable thereafter. The silk sericin-releasing bacterial nanocellulose gel showed higher moisture absorption capability

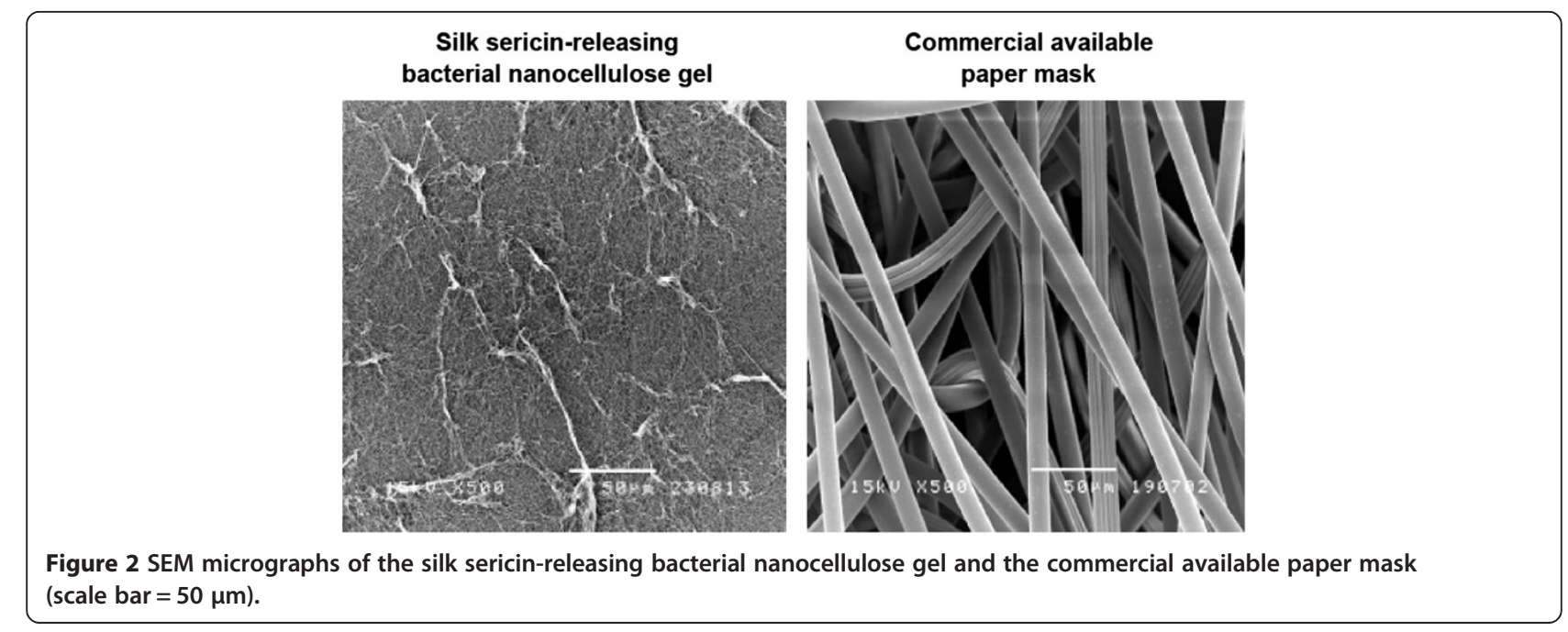


Table 2 Mechanical properties of the silk sericin-releasing bacterial nanocellulose gel and the commercial available paper mask at dry and wet conditions

\begin{tabular}{llllll}
\hline & \multicolumn{2}{l}{ Tensile modulus $\left(\mathbf{N} / \mathbf{m m}^{2}\right)$} & & & Elongation (\%) \\
\cline { 2 - 3 } \cline { 5 - 6 } & Dry & Wet & & Dry & Wet \\
\hline Silk sericin-releasing bacterial nanocellulose gel & $30.84 \pm 6.99^{*}$ & $11.23 \pm 1.26^{*}$ & & $32.89 \pm 11.89$ & $67.03 \pm 5.72^{*}$ \\
Commercial available paper mask & $0.86 \pm 0.22$ & $0.87 \pm 0.05$ & & $21.88 \pm 8.09$ & $26.9 \pm 4.38$ \\
\hline
\end{tabular}

${ }^{*}, p<0.05$ significant against the value of commercial available paper mask at corresponding condition.

than the commercially available paper mask during the incubation period. This was due to the high hydrophilicity of both bacterial nanocellulose and silk sericin $[15,16]$.

Enzymatic biodegradation rate of the silk sericin-releasing bacterial nanocellulose gel

The biodegradability of a facial mask indicates that it is bioenvironmentally friendly. The percentage of degradation of the silk sericin-releasing bacterial nanocellulose

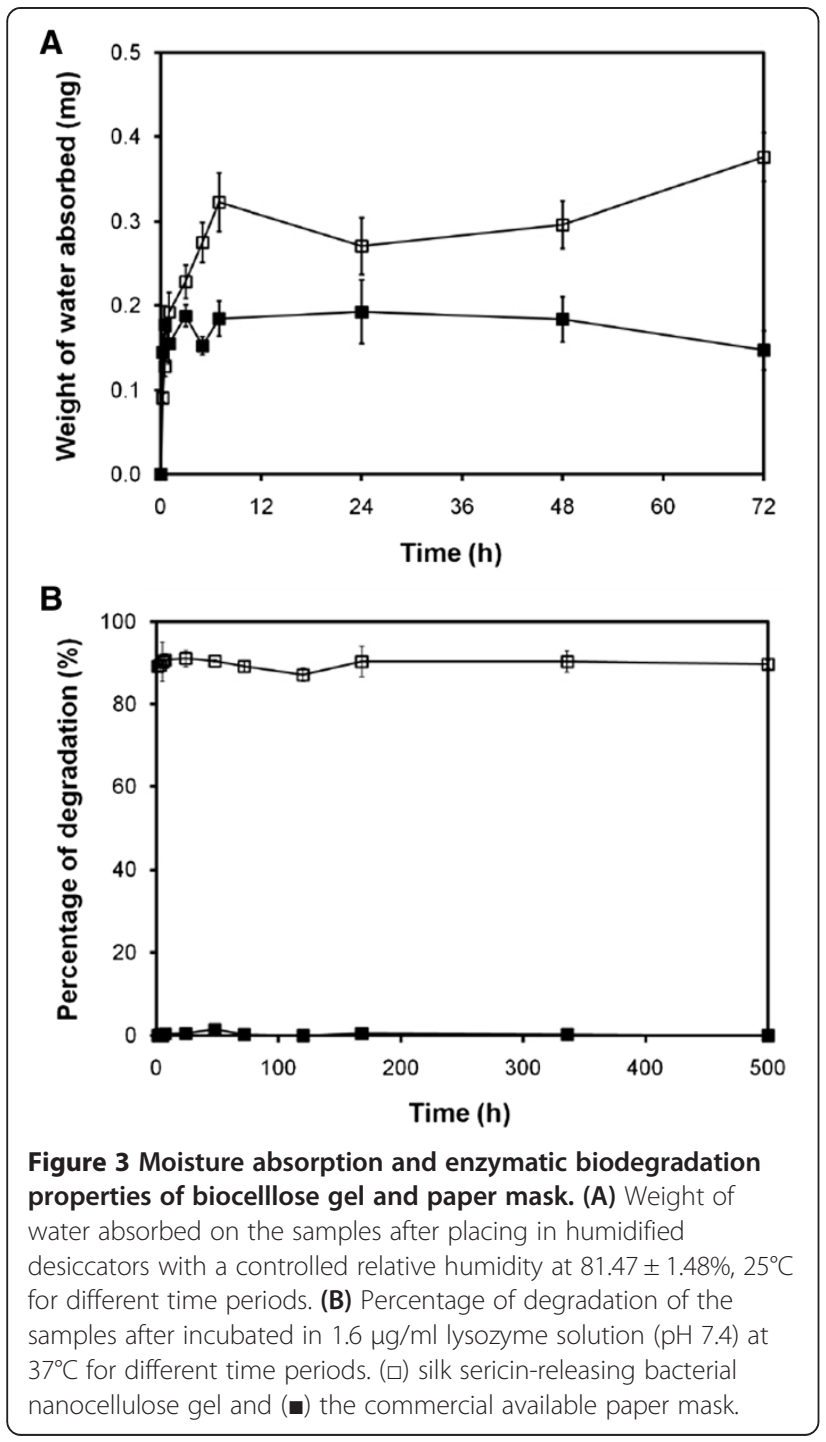

gel, compared to that of the commercially available paper mask, is illustrated in Figure 3B. A total of 90\% of the silk sericin-releasing bacterial nanocellulose gel was degraded during the incubation period. On the other hand, the commercially available paper mask was not degraded in the same condition. This would be explained by the fact that our bacterial nanocellulose gel was made of coconut water, bacteria, and silk sericin, which are all quickly biodegradable. In contrast, a commercially available paper mask is mainly cotton made of plant cellulose, waxes, and some fatty substances, which would be degraded more slowly. In addition, the plant bacterial nanocellulose of a paper mask has a mesh-like bulk work structure and tough property while our bacterial nanocellulose is more chemically pure and has a higher water-holding capacity and hydrophilicity. Thus, the bacterial nanocellulose would degrade faster than the plant bacterial nanocellulose [15].

\section{The in vitro release profile of silk sericin}

We have supposed here that the release of silk sericin from the bacterial nanocellulose gel would support the facial treatment in terms of the activation of collagen production and epithelialization of the skin [8,9]. Figure 4 demonstrates the release profile of silk sericin from the silk sericin-releasing bacterial nanocellulose gel. Initially $(0-7 \mathrm{~h})$, silk sericin was burst released from the bacterial nanocellulose gel. Later on, the release of silk sericin from the bacterial nanocellulose gel reached the plateau at $27 \%$. The release of silk sericin was possibly governed by two mechanisms: diffusion and material degradation $[17,18]$. The initial burst was the diffusional release of silk sericin absorbed on the fiber's surface. Thereafter, the silk sericin interacted with the bacterial nanocellulose fibers that were released during the degradation of the fibers. We supposed that the controlled release behavior of silk sericin from the bacterial nanocellulose gel may be beneficial for facial treatment.

\section{Adhesive property of the silk sericin-releasing bacterial nanocellulose gel}

A good facial mask should be less adhesive in order to be removed from face easily without pain. The adhesive property of the silk sericin-releasing bacterial nanocellulose gel and the commercially available paper mask was 


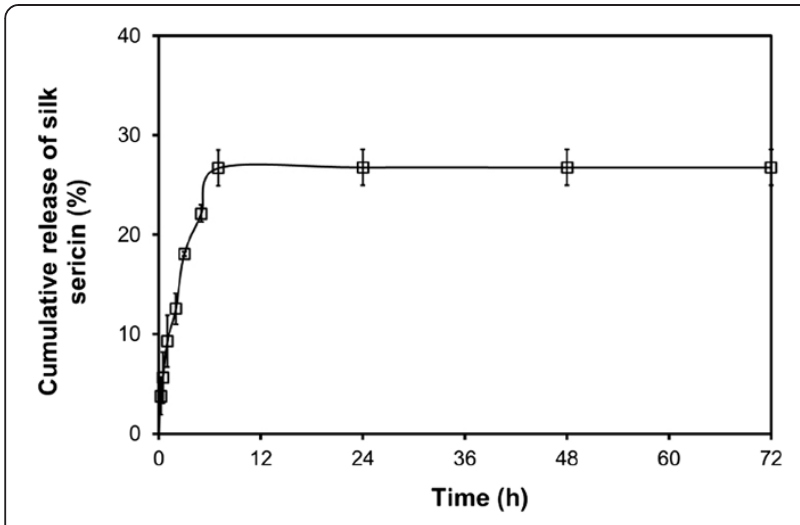

Figure 4 Percentage of cumulative release of silk sericin from the silk sericin-releasing bacterial nanocellulose gel after incubated in PBS solution ( $\mathrm{pH} 7.4$ ) at $37^{\circ} \mathrm{C}$ for different time periods.

evaluated from a number of cells on the samples after being attached to a wound on porcine skin, namely a peel assay. After being attached to the wound on porcine skin for 4 and $24 \mathrm{~h}$, the number of cells found on the silk sericin-releasing bacterial nanocellulose gel was significantly lower than that found on the commercially available paper mask (Figure 5). Therefore, the silk sericin-releasing bacterial nanocellulose gel was less adhesive than the commercially available paper mask, which may be removed more easily and with reduced pain.

\section{Non-cytotoxicity of the silk sericin-releasing bacterial nanocellulose gel}

The silk sericin-releasing bacterial nanocellulose gel must not be cytotoxic when applied as a facial mask in direct contact with facial skin. After culture by a direct contact

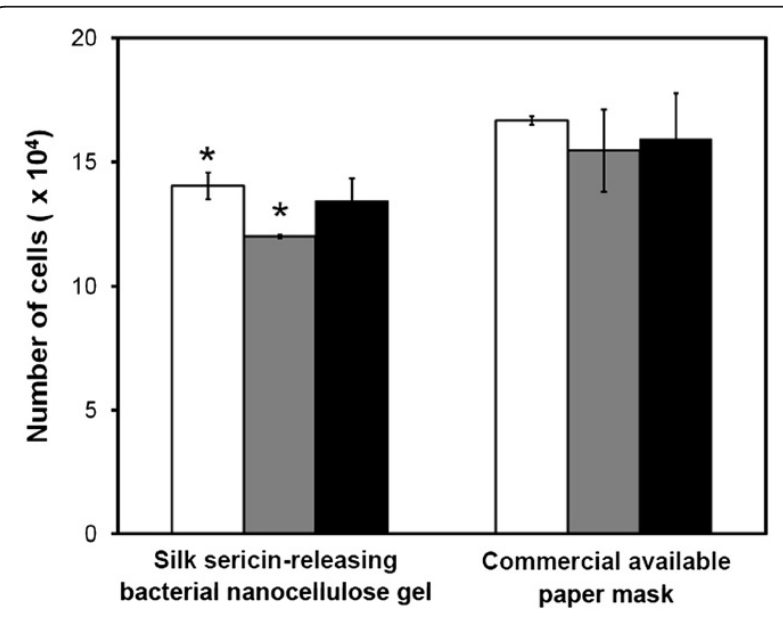

Figure $\mathbf{5}$ Number of cells on the silk sericin-releasing bacterial nanocellulose gel and the commercial available paper mask after attached to full-thickness wound of porcine skin for 4 ( $\square$ ), 24 (gray square symbol), and $48 \mathrm{~h}(\mathbf{})$. with the sample, the viable L929 cells were observed on the silk sericin-releasing bacterial nanocellulose gel, similar to those cultured on the HDPE as a negative control (Figures $6 \mathrm{~A}-\mathrm{C}$ ). In contrast, the cells were dead when cultured on rubber as a positive control (Figure 6D). Furthermore, HaCat cells cultured in the presence of silk sericin-releasing bacterial nanocellulose gel for 3-12 $\mathrm{h}$ showed as high viability $(\sim 100 \%)$ as those cultured in the presence of a commercially available paper mask (Figure 7A). The apoptotic profile of HaCat cells cultured in the presence of the sample, as analyzed by Annexin VFITC/PI double staining assay, showed that cells were both Annexin V-FITC- and PI-negative (Figure 7B). This indicated that most of the cells $(\sim 100 \%)$ were viable, but not in the apoptotic or necrotic state (Figure $7 \mathrm{C}$ ). The results confirmed that our silk sericin-releasing bacterial nanocellulose gel was non-cytotoxic.

\section{In vivo safety of the silk sericin-releasing bacterial nanocellulose gel}

To prove its safety for cosmetic application, the silk sericin-releasing bacterial nanocellulose gel was subcutaneously implanted into rats and the tissue responses were evaluated according to ISO10993-6 standard (Biological evaluation of medical devices - Part 6: Tests for local effects after implantation) in comparison to the commercially available paper mask. The rats that received the implantation of both samples were healthy throughout the implantation period. No signs of inflammation (i.e. redness, swelling, pain, and heat) were observed in any of the rats. Figure 8 shows images of the H\&E-stained sections of silk sericin-releasing bacterial nanocellulose gel and the commercially available paper mask implanted. The arrows indicate the interface between the implanted sample and surrounding tissue. No excessive inflammatory reaction was detected around the implantation sites. The infiltration of inflammatory cells into the implanted samples is shown in Figure 9. The number of inflammatory cells that infiltrated into the silk sericin-releasing bacterial nanocellulose gel was comparable to that of the commercially available paper mask during the implantation period. The intensity of inflammatory cells, necrosis, fibrosis, neovascularization, and fatty infiltrate was graded as presented in Table 3. It was observed that polymorphonuclear cells were packed and infiltrated into silk sericin-releasing bacterial nanocellulose gel after 3 and 7 weeks of implantation. However, its intensity was reduced thereafter. Lymphocytes, macrophages, and neovascularization were also found in both samples during the implantation period. The implanted commercially available paper mask showed more intensity of fatty infiltrate and giant cells than the implanted silk sericin-releasing bacterial nanocellulose gel. On the other hand, plasma cells and necrosis were not observed in either sample during the implantation period. 

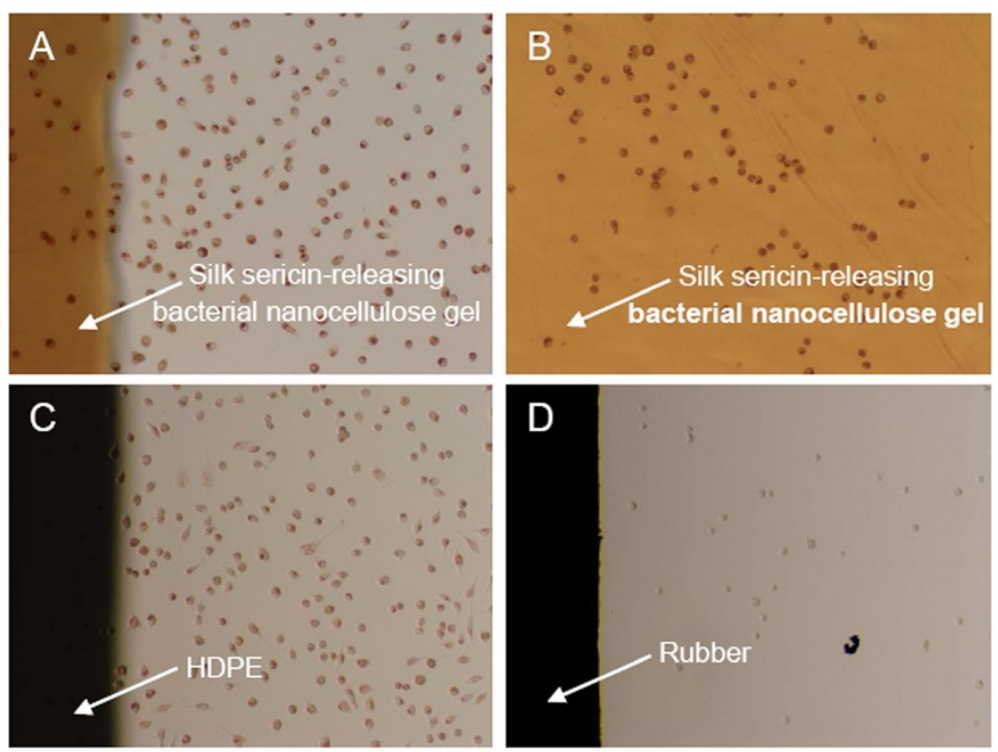

Figure 6 Morphology of L929 mouse fibroblast cells after cultured on materials for $\mathbf{4 8}$ h. (A,B) silk sericin-releasing bacterial nanocellulose gel, (C) high-density polyethylene (HDPE, negative control), and (D) rubber (positive control).

To sum up, the implantation of silk sericin-releasing bacterial nanocellulose gel was evaluated as being nonirritant, relative to the commercially available paper mask. This confirmed that our silk sericin-releasing bacterial nanocellulose gel was safe for further investigation in the application of facial treatment.

\section{Conclusion}

Silk sericin-releasing bacterial nanocellulose gel has been developed in this study to be applied as a bioactive mask for facial treatment. We found that a $\mathrm{pH}$ of 4.5 was optimal for the production of the bacterial nanocellulose gel, resulting in a $0.6 \mathrm{~cm}$ soft and stable gel with a smooth surface. To improve the biological properties, the gel was absorbed with silk sericin. The silk sericin-releasing bacterial nanocellulose gel had an ultrafine and extremely pure fiber network structure. The mechanical properties and moisture absorption ability of the silk sericin-releasing bacterial nanocellulose gel were improved, compared to those of the commercially available paper mask. Silk sericin could be control-released from the gel. Furthermore, the silk sericin-releasing bacterial nanocellulose gel was less adhesive and non-cytotoxic, compared to the commercially available paper mask. The in vivo safety test confirmed that our silk sericin-releasing bacterial nanocellulose gel was nonirritant. Further investigation into this novel bioactive facial mask has been carried out in clinic. We supposed that the silk sericin-releasing bacterial nanocellulose gel would be an outstanding candidate in the application of facial treatment.

\section{Methods}

Materials

Coconut water was obtained from coconuts purchased locally. Ammonium sulfate $\left(\left(\mathrm{NH}_{4}\right)_{2} \mathrm{PO}_{4}\right)$, glacial acetic acid $\left(\mathrm{CH}_{3} \mathrm{COOH}\right)$, and other chemicals used were of analytical grade purchased from Sigma-Aldrich, USA. All chemicals were used without further purification.

\section{Organism}

The G. xylinus strain (ATCC 23769) was isolated from nata de coca and was supplied by Kasetsart University, Bangkok, Thailand.

\section{Culture medium}

The coconut water sucrose medium as reported in the work of Verschuren et al. was used in this study with a slight modification [19]. The initial $\mathrm{pH}$ of sucrose medium was adjusted to $3.0,3.5,4.0,4.5$, or 5.0 with $\mathrm{CH}_{3} \mathrm{COOH}$. Then, $10 \mathrm{~mL}$ of the G. xylinus strain at $10^{5} \mathrm{cfu} / \mathrm{mL}$ was added into the $\mathrm{pH}$-adjusted coconut water sucrose medium and poured into tissue culture plates for further fermentation process.

\section{Fermentation process}

For each $\mathrm{pH}$ value of the coconut water sucrose medium, the fermentation was incubated at $30^{\circ} \mathrm{C}$ under static conditions for 7-10 days until the bacterial nanocellulose gels were formed completely. The formed bacterial nanocellulose gels were washed with $2 \%$ aqueous $\mathrm{NaOH}$ solution at $70^{\circ} \mathrm{C}$ for $10 \mathrm{~min}$, and then washed repeatedly until a 

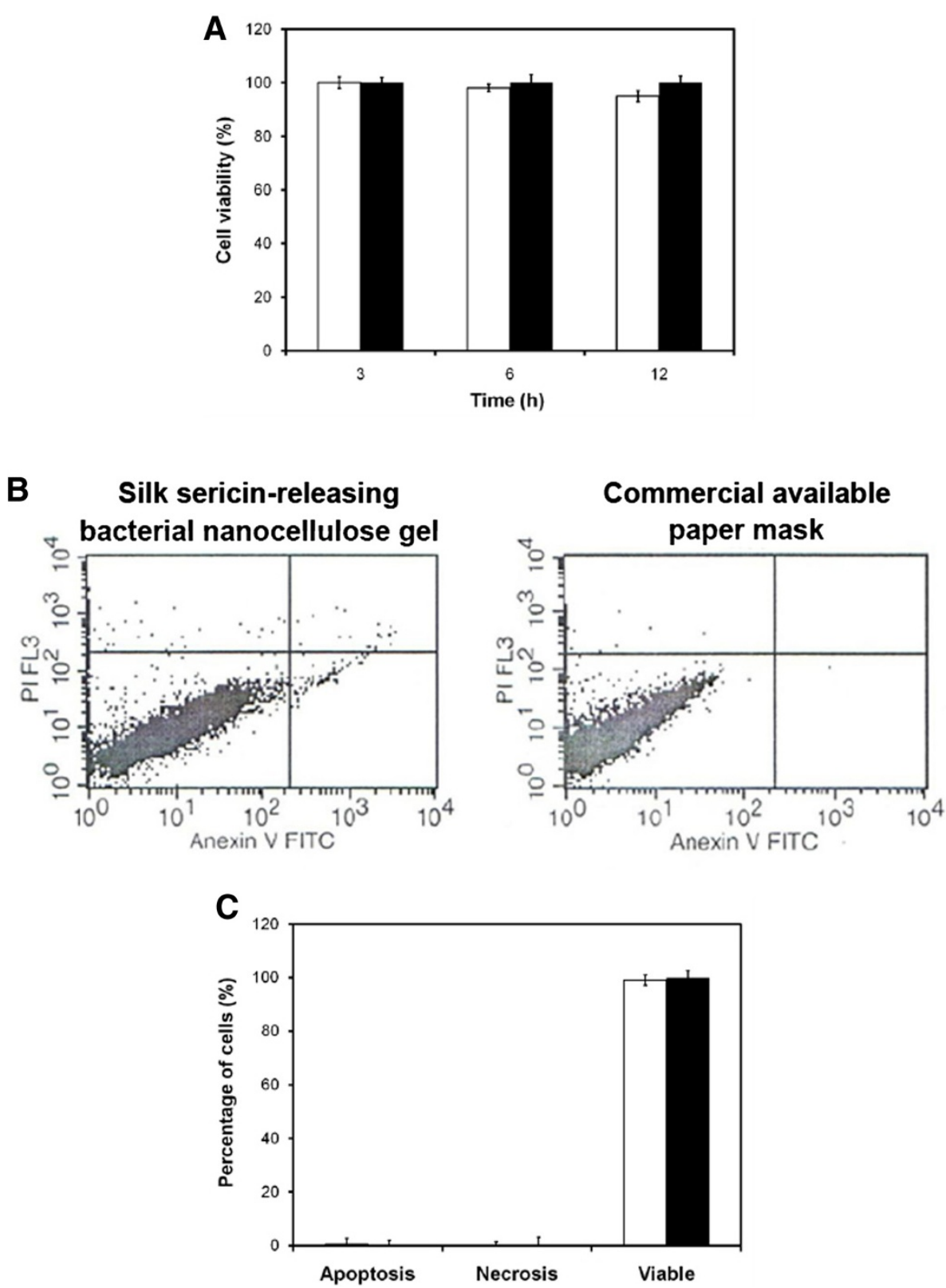

Figure 7 Non-cytotoxicity of silk sericin-releasing bacterial nanocellulose gel and paper mask. (A) Percentage of viability of HaCat cells cultured in the presence of samples for 3, 6, and 12 h. (B) Apoptotic profile and (C) Viability percentage of HaCat cells cultured in the presence of samples for $3 \mathrm{~h}$, analyzed by Annexin V-FITC/PI double staining assay. (口) silk sericin-releasing bacterial nanocellulose gel (घ) and the commercial available paper mask.

neutral $\mathrm{pH}$ was obtained. The bacterial nanocellulose gel was sterilized by gamma radiation before the experiment. Silk sericin solution $(0.1 \mathrm{~g} / \mathrm{mL})$ was prepared in deionized water. The silk sericin solution $(0.2 \mathrm{~mL})$ was absorbed into the bacterial nanocellulose gel (diameter $3.5 \mathrm{~cm}$ ) and incubated at $25^{\circ} \mathrm{C}$ for $0.5 \mathrm{~h}$ to allow the silk sericin impregnated into the surface of bacterial nanocellulose gel. The silk sericin-releasing bacterial nanocellulose gel was obtained.

\section{Morphological observation and thickness measurement}

The internal structure of samples was observed on a scanning electron microscope (SEM, JSM 5400, JEOL) at an accelerating voltage of $12-15 \mathrm{kV}$ after sputtercoating with gold. The thickness of the bacterial nanocellulose gels prepared from different $\mathrm{pH}$ was measured using ImageJ software (the US National Institutes of Health, USA). 


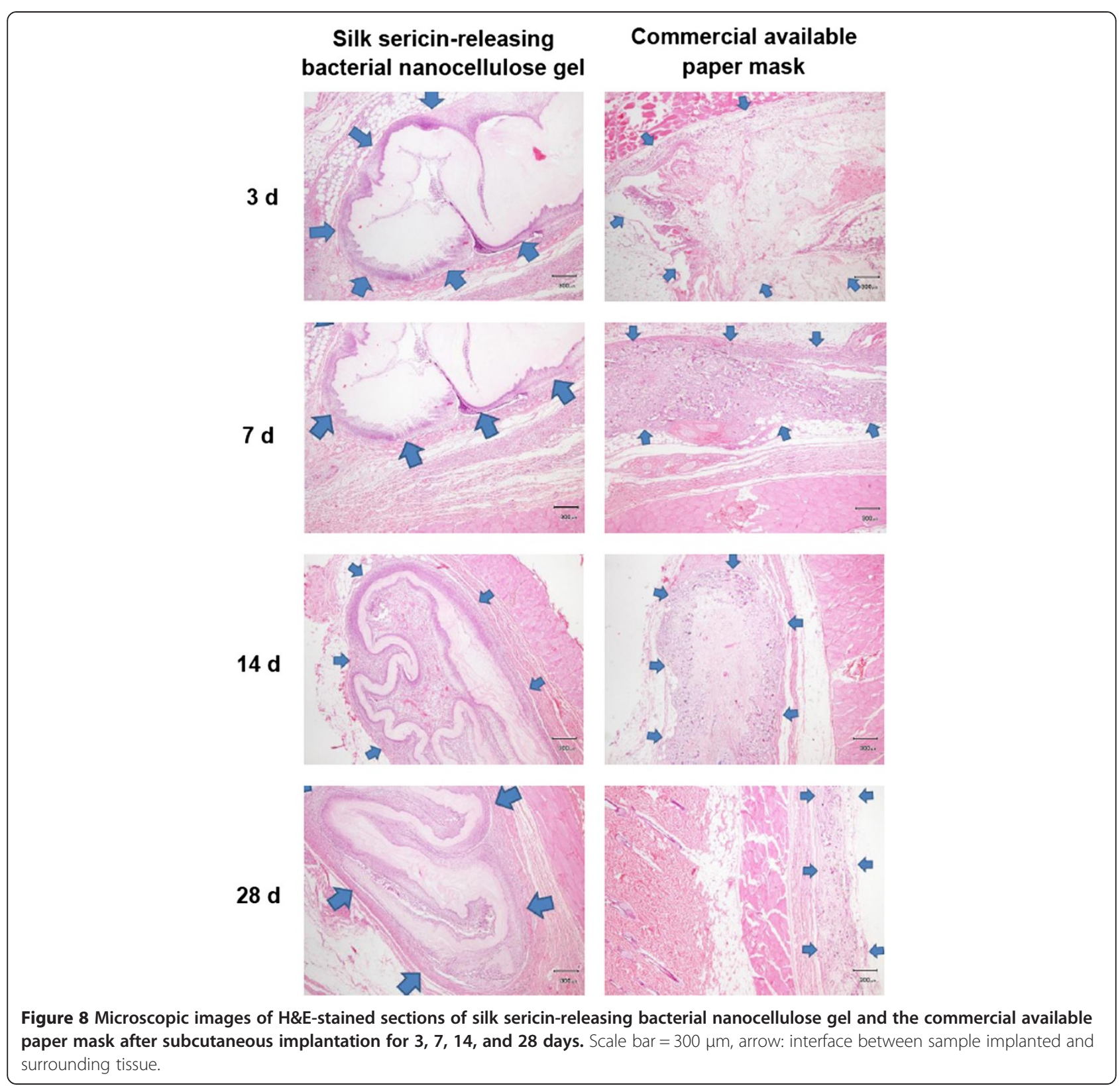

\section{Mechanical test}

A tensile test was performed on the wet samples $(\mathrm{L}=150 \mathrm{~mm}, \mathrm{~W}=25 \mathrm{~mm}, \mathrm{H}=3 \mathrm{~mm})$ at room temperature using a universal testing machine (Instron, No. 5567 ) at a constant rate of $300 \mathrm{~mm} / \mathrm{min}$. The force curves as a function of deformation $(\mathrm{mm})$ were automatically recorded by the software. The tensile modulus and elongation at break were calculated according to the ASTM D638-01 method $(n=6)$.

\section{Moisture absorption test}

The moisture absorption capability of the samples was evaluated by placing the dried sample in the desiccators in which the relative humidity was controlled by salt solution. Potassium chloride was used to obtain a relative humidity of $81.47 \pm 1.48 \%$ at $25^{\circ} \mathrm{C}$ [20]. The sample was removed from the desiccators at the predetermined times and carefully weighed. The percentage of weight increase of the sample was calculated relative to their initial weights $(n=6)$.

\section{In vitro enzymatic biodegradation test}

A known weight sample was incubated in $1.6 \mu \mathrm{g} / \mathrm{mL}$ of lysozyme solution ( $\mathrm{pH} 7.4)$ at $37^{\circ} \mathrm{C}$. The enzyme solution was changed every 2 days to ensure continuous enzyme activity. At each time interval, the remaining 


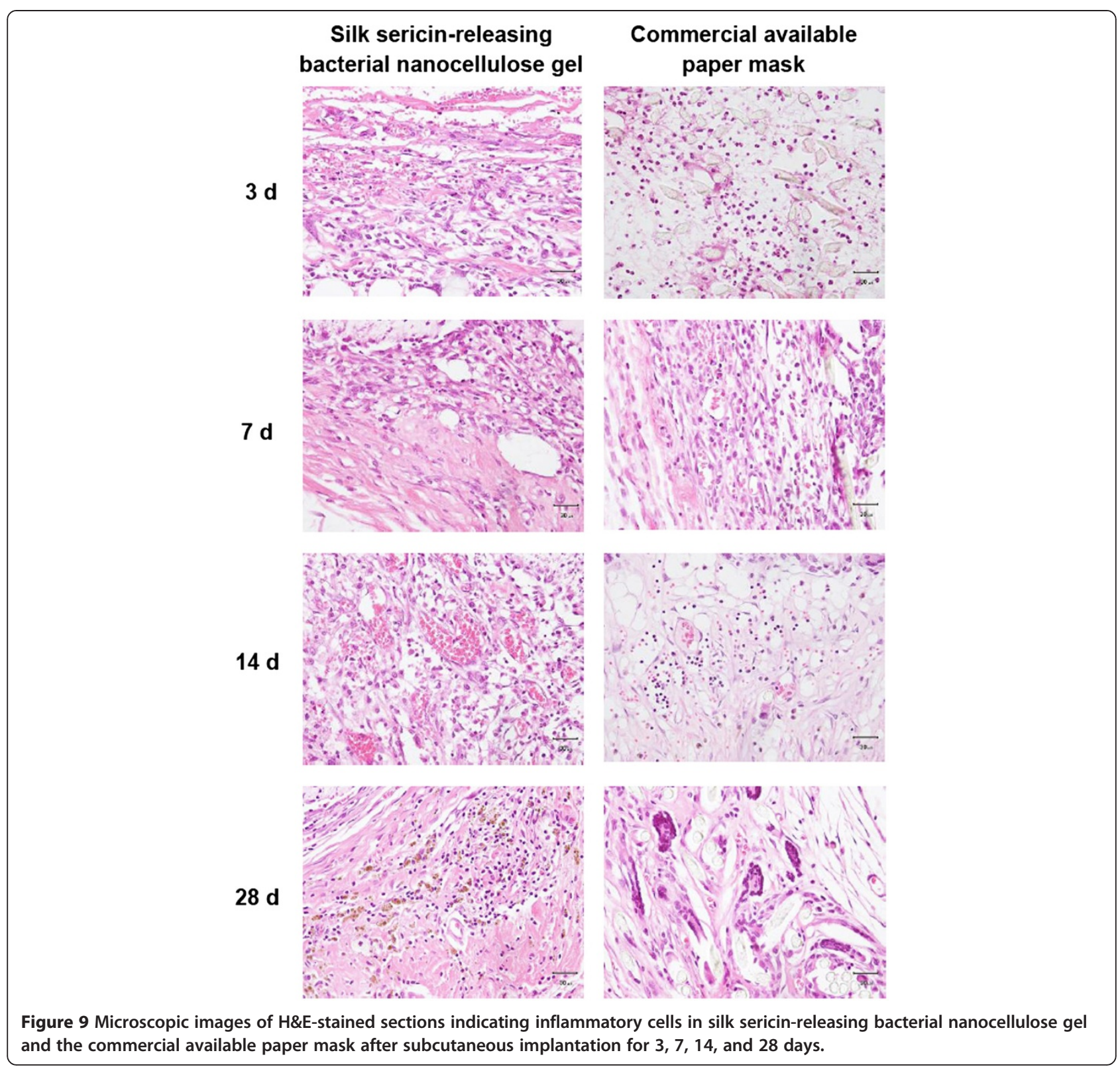

sample was taken out of the solution, rinsed repeatedly with deionized water, and freeze dried. The dried sample was weighed and the percentage of weight loss was calculated as follows:

$$
\text { Percentage of weight loss }(\%)=\left(\frac{W_{0}-W_{t}}{W_{0}}\right) \times 100
$$

Where $W_{O}$ and $W_{t}$ represent the initial weight of the sample before degradation and the weight of the sample after degradation at different time periods, respectively $(\mathrm{n}=3)$.

\section{In vitro release test of silk sericin}

The samples were placed into phosphate-buffered saline solutions (PBS, $\mathrm{pH} 7.4$ ) at $37^{\circ} \mathrm{C}$ with a continuous stirring in a closed container. The PBS solutions $(1.5 \mathrm{~mL})$ were collected at the predetermined times and the amount of silk sericin released into the solution was measured using a BCA protein assay kit (Pierce, Rockford, IL, USA). The absorbance of the solution was measured at $562 \mathrm{~nm}$ and the amount of silk sericin was determined from a standard 
Table 3 Average intensity of inflammatory cells, necrosis, fibrosis, neovascularization, and fatty in silk sericinreleasing bacterial nanocellulose gel and the commercial available paper mask after subcutaneous implantation for $3,7,14$, and 28 days

\begin{tabular}{|c|c|c|c|c|c|c|c|c|}
\hline & \multicolumn{2}{|c|}{3 days } & \multicolumn{2}{|c|}{7 days } & \multicolumn{2}{|c|}{14 days } & \multicolumn{2}{|c|}{28 days } \\
\hline & $\mathrm{B}^{*}$ & $\mathrm{C}^{* *}$ & B & $\mathrm{C}$ & B & C & B & $\mathrm{C}$ \\
\hline PMN & $4+$ & 2.25 & 4 & 1.75 & 3.25 & 1.75 & 3.25 & 1 \\
\hline Lymphocytes & 1 & 2 & 2 & 2 & 2.75 & 2 & 2.5 & 1.75 \\
\hline Plasma cells & 0 & 0 & 0 & 0 & 0 & 0 & 0 & 0 \\
\hline Macrophages & 3 & 2.5 & 3 & 3 & 3 & 3 & 3 & 3 \\
\hline Giant cells & 0 & 0.5 & 0 & 2 & 0.75 & 3 & 1 & 3 \\
\hline Necrosis & 0 & 0 & 0 & 0 & 0 & 0 & 0 & 0 \\
\hline Fibrosis & 4 & 2.75 & 4 & 3.25 & 4 & 4 & 4 & 3.5 \\
\hline Neovascularization & 3 & 2.5 & 3 & 1.25 & 2.75 & 1.75 & 2 & 3 \\
\hline Fatty infiltrate & 0 & 3 & 0 & 1 & 0 & 0.75 & 0 & 0.5 \\
\hline
\end{tabular}

*B: silk sericin-releasing bacterial nanocellulose gel.

${ }^{* *} \mathrm{C}$ : commercial available paper mask.

† Intensity 0-4: $0=$ not observed, $1=$ rare, $2=$ minimal, $3=$ heavily infiltrate and $4=$ packed infiltrate.

curve prepared from different concentrations of bovine serum albumin $(\mathrm{n}=3)$.

\section{Peel test with porcine skin}

Porcine skin was used within $2 \mathrm{~h}$ after sacrifice. Fullthickness wounds were prepared by cutting the skin to a depth of $1 \mathrm{~cm}$. The samples were attached to the wound. After 4, 24, and $48 \mathrm{~h}$, the sample was removed and the number of cells attached to the sample was analyzed by the fluorometric quantification of cellular DNA according to the method reported by Takahashi et al. $(\mathrm{n}=3)$ [21].

\section{Cytotoxic test with L929 mouse fibroblast cells}

A cytotoxic test of the sample was performed using a direct contact assay. High-density polyethylene (HDPE) and natural rubber containing carbon black were used as negative and positive controls, respectively. L929 mouse fibroblast cells (ECACC no. 85011425) were seeded onto the sterilized samples $\left(1 \times 1 \mathrm{~cm}^{2}\right)$ at a density of $6 \times 10^{4}$ cells/sample and cultured in Dulbecco's Modified Eagle Medium (DMEM) supplemented with 10\% (v/v) Fetal Bovine Serum (FBS) and $100 \mathrm{U} / \mathrm{mL}$ of penicillin/streptomycin at $37^{\circ} \mathrm{C}, 5 \% \mathrm{CO}_{2}$. After $48 \mathrm{~h}$ of culture, the cells were stained with $0.01 \%(\mathrm{w} / \mathrm{v})$ neutral red solution ( $\mathrm{pH}$ 7.4). The morphology of the cells was observed on a phase contrast microscope.

\section{Viability test with $\mathrm{HaCaT}$ human keratinocyte cells}

$\mathrm{HaCaT}$ human keratinocyte cells were seeded onto a 96well plate at a density of $5 \times 10^{3}$ cells/well and cultured in DMEM supplemented with 10\% (v/v) FBS and $100 \mathrm{U} /$ $\mathrm{mL}$ of penicillin/streptomycin at $37^{\circ} \mathrm{C}, 5 \% \mathrm{CO}_{2}$. After
$24 \mathrm{~h}$ of cell attachment, the medium was refreshed and the sterilized sample was placed into the medium. After 3,6 , and $12 \mathrm{~h}$ of culture in the presence of the sample, the number of cells was quantified using the conventional 3-(4,5-dimethylthiazol-2-yl)-2,5-diphenyltetra zolium bromide (MTT) assay [22]. The cells cultured on the plate in the presence of a commercially available paper mask in the same conditions were used as a control group $(\mathrm{n}=3)$.

\section{Cell apoptotic test by Annexin V-FITC/PI double staining assay}

$\mathrm{HaCaT}$ human keratinocyte cells were cultured in the presence of samples for $3 \mathrm{~h}$, as previously described. Apoptosismediated cell death was examined using a double staining method with a fluorescein isothiocyanate (FITC)-labeled Annexin V/propidium iodide (PI) Apoptosis Detection kit (BD Pharmingen ${ }^{\mathrm{TM}}$, BD Biosciences, USA) according to the manufacturer's instructions. Flow cytometric analysis was performed immediately after staining. Cells in the early stages of apoptosis were Annexin-V-FITC-positive, whereas cells that were both Annexin V-FITC and PI-positive were in the late stages of apoptosis.

\section{In vivo safety test (ISO10993-6 Standard)}

An in vivo test was approved by the Ethics Committee of the Faculty of Medicine, Chulalongkorn University. The animal experiments were performed according to the Chulalongkorn University Animal Care and Use Committee (CU-ACUC) under standard sterile conditions. The implantation of silk sericin-releasing bacterial nanocellulose gel and the commercially available paper mask $\left(2 \times 2 \mathrm{~cm}^{2}\right)$ into the subcutaneous tissue of female Wistar rats (8 weeks old, 200-300 g) was carried out. Briefly, the rats were anesthetized, and their hair was shaved, and disinfected with $70 \mathrm{vol} \%$ ethyl alcohol. A $1 \mathrm{~cm}$ skin incision was made to form pockets in the subcutaneous tissue, then the sample was inserted into each pocket. The wound was closed with $6-0$ prolene suture and disinfected with Betadine ${ }^{\bullet}$ (povidone-iodine topical antiseptics) solution. After 3, 7, 14, and 28 days of implantation, the rats were sacrificed with an overdose of thiopental sodium. The samples and surrounding tissue were retrieved, fixed with $10 \mathrm{vol} \%$ formalin solution, and embedded in paraffin. The paraffin-embedded samples were sectioned and stained with hematoxylin and eosin (H\&E). For histological assessment, the H\&E slides were semi-quantitatively scored following ISO10993-6. Inflammatory cell types, neovascularization, fibrosis, and fatty infiltrate were scored by one pathologist at two different times. The intensity of inflammatory cells, neovascularization, fibrosis, and fatty infiltrate was recorded using 0-4 scales $(0=$ not observed, $1=$ rare, $2=$ minimal, $3=$ heavily infiltrated, and $4=$ packed infiltrated). The final score was calculated according to Equation 2 and classified as follows: 
$0.0-2.9$ (sample is nonirritant), 3.0-8.9 (sample is slightirritant), $9.0-15$ (sample is moderate-irritant), and $>15$ (sample is severe-irritant). The level of irritation was compared to that of the commercially available paper mask as a control sample.

$$
\text { Final score }=\left[2 I_{t}+N_{t}\right]-\left[2 I_{c}+N_{c}\right]
$$

where $I_{i}$ is the total number of polymorphonuclear cells, lymphocytes, plasma cells, macrophages, giant cells, and necrosis of sample $i(i=$ test sample $(t)$ and control $(c)), N_{i}$ is the total number of neovascularization, fibrosis, and fatty infiltrate of sample $i(i=$ test sample $(t)$ and control $(c))$.

\section{Statistical analysis}

All quantitative data represent the mean \pm standard deviation. The statistical significance was determined by paired and unpaired Student's t-tests and ANOVA. A value of $p<0.05$ was considered significant.

\section{Competing interests}

The authors declare that they have no competing interests.

\section{Authors' contributions}

PA conceived of the study, and participated in its design and coordination. NB carried out the experiments. Both authors participated in experimental designs, data analyses, read and approved the final manuscript.

\section{Acknowledgement}

This research was supported by the Ratchadapiseksompoch Endowment Fund (2014), Chulalongkorn University (CU-57-091-IC).

Received: 14 May 2014 Accepted: 27 November 2014

Published online: 09 December 2014

\section{References}

1. Updegraff DM: Semimicro determination of cellulose in biological materials. Anal Biochem 1969, 32:420-424

2. Ruka DR, Simon GP, Dean KM: Altering the growth conditions of Gluconacetobacter xylinus to maximize the yield of bacterial cellulose. Carbohyd Polym 2012, 89:613-622.

3. Lin WC, Lien CC, Yeh HJ, Yu CM, Hsu SH: Bacterial cellulose and bacterial cellulose-chitosan membranes for wound dressing applications. Carbohyd Polym 2013, 94:603-611.

4. Kurosumi A, Sasaki C, Yamashita Y, Nakamura Y: Utilization various fruit juices as carbon source for production of bacterial cellulose by Acetobacter xylinum NBRC 13693. Carbohyd Polym 2009, 76:333-335.

5. Kongruang S: Bacterial cellulose production by Acetobacter xylinum strains from agricultural waste products. Appl Biochem Biotechnol 2008, 148:245-256.

6. Cuttle L, Kempf M, Phillips GE, Mill J, Hayes MT, Fraser JF, Wang XQ, Kimble RM: A porcine deep dermal partial thickness burn model with hypertrophic scarring. Burns 2006, 32:806-820

7. Gibran NS, Boyce S, Greenhalgh DG: Cutaneous wound healing. J Burn Care Res 2007, 28:577-579.

8. Aramwit $P$, Sangcakul A: The effects of sericin cream on wound healing in rats. Biosci Biotechnol Biochem 2007, 71:2473-2477.

9. Aramwit P, Kanokpanont S, De-Eknamkul W, Kamei K, Srichana T: The effect of sericin with variable amino-acid content from different silk strains on the production of collagen and nitric oxide. J Biomater Sci Polym Ed 2009, 20:1295-1306.

10. Aramwit $P$, Kanokpanont $S$, Nakpheng $T$, Srichana $T$ : The effect of sericin from various extraction methods on cell viability and collagen production. Int J Mol Sci 2010, 11:2200-2211.
11. Vandamme EJ, Baets SD, Vanbaelen A, Joris K, Wulf PD: Improved production of bacterial cellulose and its application potential. Polym Degrad Stab 1998, 59:93-99.

12. Jonas R, Farah Luiz F: Production and application of microbial cellulose. Polym Degrad Stab 1998, 59:101-106.

13. Kanokpanont S, Damrongsakkul S, Ratanavaraporn J, Aramwit P: Physicochemical properties and efficacy of silk fibroin fabric coated with different waxes as wound dressing. Int J Biol Macromol 2013, 55:88-97.

14. Wongputtaraksa T, Ratanavaraporn J, Pichyangkura R, Damrongsakkul S: Surface modification of Thai silk fibroin scaffolds with gelatin and chitooligosaccharide for enhanced osteogenic differentiation of bone marrow-derived mesenchymal stem cells. J Biomed MatEr Res Part B 2012, 100B:2307-2315.

15. Klemm D, Schumann D, Udhardt U, Marsch S: Bacterial synthesized cellulose - artificial blood vessels for microsurgery. Prog Polym Sci 2001, 26:1561-1603

16. Zhang YQ: Applications of natural silk protein sericin in biomaterials. Biotechnol Adv 2002, 20:91-100

17. Ratanavaraporn J, Furuya $H$, Kohara H, Tabata $Y$ : Synergistic effects of the dual release of stromal cell-derived factor- 1 and bone morphogenetic protein-2 from hydrogels on bone regeneration. Biomaterials 2011, 32:2797-2811.

18. Simmons CA, Alsberg E, Hsiong S, Kim WJ, Mooney DJ: Dual growth factor delivery and controlled scaffold degradation enhance in vivo bone formation by transplanted bone marrow stromal cells. Bone 2004, 35:562-569.

19. Verschuren PG, Cardona TD, Nout MJR, Gooijer KDD, Van Den Heuvel JC: Location and limitation of cellulose production by Acetobacter xylinum established from oxygen profiles. J Biosci Bioeng 2000, 89:414-419.

20. Greenspan L: Humidity fixed points of binary saturated aqueous solutions. Physics 1977, 81A:89-96.

21. Takahashi $Y$, Yamamoto M, Tabata Y: Osteogenic differentiation of mesenchymal stem cells in biodegradable sponges composed of gelatin and $\beta$-tricalcium phosphate. Biomaterials 2005, 26:3587-3596.

22. Mosmann T: Rapid colorimetric assay for cellular growth and survival: Application to proliferation and cytotoxicity assays. $J$ Immunol Methods 1983, 65:55-63.

doi:10.1186/s12896-014-0104-x

Cite this article as: Aramwit and Bang: The characteristics of bacterial nanocellulose gel releasing silk sericin for facial treatment. $B M C$

Biotechnology 2014 14:104.

\section{Submit your next manuscript to BioMed Central and take full advantage of:}

- Convenient online submission

- Thorough peer review

- No space constraints or color figure charges

- Immediate publication on acceptance

- Inclusion in PubMed, CAS, Scopus and Google Scholar

- Research which is freely available for redistribution 